\title{
Transparent and ultra-lightweight design for ultra-broadband asymmetric transmission of airborne sound
}

\author{
Jie $\mathrm{Hu}^{1,2, *}$, Bin Liang ${ }^{1}$, and Xiaojun Qiu ${ }^{3}$ \\ ${ }^{1}$ Key Laboratory of Modern Acoustics (MOE), Institute of Acoustics, Nanjing University, Nanjing, \\ China \\ ${ }^{2}$ College of information Science and Technology, Nanjing Forestry University, Nanjing, China \\ ${ }^{3}$ Centre for Audio, Acoustics and Vibration, Faculty of Engineering and IT, University of Technology \\ Sydney, Sydney, Australia
}

\section{ABSTRACT}

The past few years have witnessed considerable efforts dedicated to the design of functional devices capable of producing asymmetric transmission for sound which may have far-reaching impact on diverse applications. However the existing designs usually rely on various artificial materials that yield special acoustical parameters based on resonance effects and therefore have limited bandwidth and complicated structures. This poses challenges for practical applications. Here we propose to break through the limit in bandwidth by designing an ultra-lightweight and optically transparent structure with asymmetric transmission property. Instead of fabricating solids into complex resonators to build artificial materials for airborne sound that is inevitably limited by bandwidth and weight, we simply use xenon to fill a spatial region of an asymmetric shape which allows the incident plane wave to pass along one particular direction while provides nearly total reflection when the incident direction is reversed regardless of its frequency. The effectiveness of the proposed design is verified via numerical simulations, and the results show that a highly asymmetric transmission of airborne sound persists within a ultra-broad frequency range. The realization of high efficiency and broadband asymmetric acoustic manipulation with a simple, ultra-lightweight and optically transparent structure offers new possibility for the design of

\footnotetext{
*Email: hujie@njfu.edu.cn
} 
acoustic one-way devices and may have great application potential in a variety of scenarios such as noise control.

Keywords: asymmetric transmission, refraction, broadband.

Introduction

Recent emergence of the acoustic diode, as the acoustic counterpart of electrical diode, gives rise to one-way manipulation of acoustic waves that are conventionally believed always transmit symmetrically in a given path. During the past few years, asymmetric transmission of acoustic waves have attracted considerable attentions due to the potential of such special kinds of devices to lead to revolutionary changes in various acoustic applications. Compared with the original design of acoustic one-way devices that only have low performance as a result of using nonlinearity to break through the limit of reciprocity [1,2], many schemes have been proposed with substantial improvements in performance in linear regime. Thanks to the development of different mechanisms for achieving asymmetric transmission with linear structures such as that using asymmetric mode conversion, these designs are endowed with remarkable enhancement in terms of efficiency, bandwidth, thinness, etc. [3-6], or with some special functionalities such as keeping the acoustic path open to light and heat [7].

However, the design and fabrication of acoustic metamaterials usually involve many difficulties due to their subwavelength nature, and the introduction of solids for building metamaterial units unavoidably increase the weight dramatically in comparison with air as background medium. Furthermore, the working bandwidth of the metamaterials based one-way devices are still limited even for those composed of non-resonant units because they cannot work at high frequency 
regime where the effective medium approximation fails. Considering the large range of the spectrum of practical acoustic signals, it is highly desired to unveil new mechanism for realizing asymmetric acoustic transmission without being restricted by the bandwidth.

In this work, we make an attempt to address this issue by designing a structure capable of allowing the transmission of airborne sound only along one particular direction with theoretically unlimited bandwidth. The mechanism is that, instead of relying on artificial materials with limited bandwidth and complicated configuration, we simply use a natural material with innate dispersionless property to construct a polygon-shape model that have interfaces with different angles for incident wave coming from the two opposite sides, regardless of the driving frequency. By judiciously designing the structural parameters of this structure, it becomes possible that the normally incident wave will be subject to total reflection only in one direction but be guided to transmit through the system along a controlled direction in the reversed direction. Furthermore, this material as inclusion can be chosen as another gas with high refractive indexes and low mass density, ensuring ultra-lightweight and transparent features of the resulting devices. We use analytical analysis as well as numerical simulations to demonstrate our mechanism as follows.

\section{Theory}

First we explain the mechanism underlying our scheme for producing ultra-broadband asymmetric transmission with an ultra-lightweight and optically transparent structure. For simplicity without losing generality, we consider two-dimensional case in the current study. Figure 1 schematically illustrates the proposed structure that is simply formed by filling a polygonal region with a specific kinds of gas with higher refractive index than the background medium (air), 
which is chosen as xenon here. The sound velocity and the normalized refractive index of air are $c_{\mathrm{A}}=340 \mathrm{~m} / \mathrm{s}$ and $n_{\mathrm{A}}=1$ respectively, and the acoustical parameters of xenon are $c_{\mathrm{X}}=169 \mathrm{~m} / \mathrm{s}$ and $n_{\mathrm{X}}=2.03$ respectively. The designed structure is situated inside a straight waveguide with hard boundaries on both sides for a better guidance of the incident plane wave. The two different kinds of gases, i.e., air and xenon, are separated by inserting lightweight thermoplastic films in between which are sufficiently thin so that one could treat them as acoustically transparent layers.
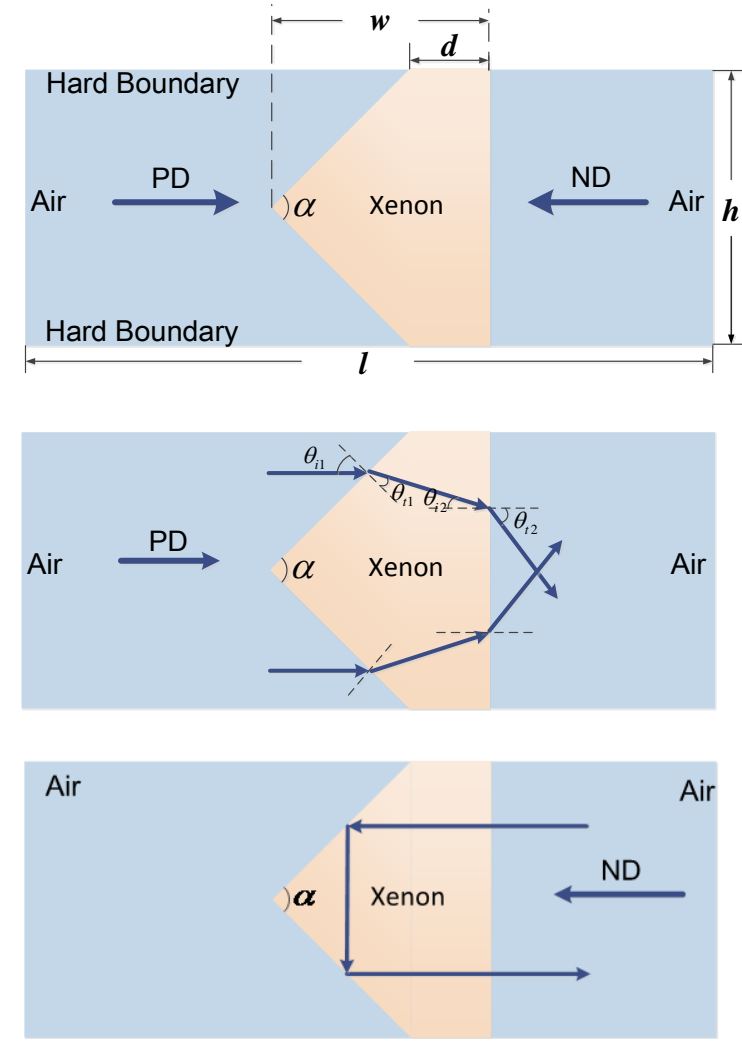

FIG 1 The schematic of our proposed structure with asymmetric transmission property of airborne sound, which has a polygonal shape with a vertex at the left side and a plane at the right side for producing refraction and total reflection for plane waves coming from the two opposite sides respectively.

The shape of the whole structure is a convex polygon, with the interior angle of the leftmost vertex being $\alpha$ and the right side being a plane vertical to the waveguide boundary. The 
structural parameters of the designed model are $h=0.3 \mathrm{~m}, l=1.0 \mathrm{~m}, d=0.09 \mathrm{~m}$ and $w=0.24 \mathrm{~m}$, respectively, with the parameters of $h, l, d$ and $w$ referring to the height of the structure, the length of the structure, the length of the upper and lower polygon sides and the maximum width of the whole polygon, respectively. This polygonal structure is designed for having an asymmetric transmission property for plane acoustic waves normally incident from the two opposite sides, i.e., propagate along the axis of the waveguide from left and right respectively, as will be demonstrated both theoretically and numerically in what follows. In the current study we choose the vertex angle at the left side as a right angle (viz., $\alpha=90^{\circ}$ ). It is worth mentioning that, however, our scheme is universal and can yield different propagation directions for the transmitted wave by tailoring the value of $\alpha$.

The mechanism of our proposed structure can be understood as follows. Acoustic wave will reflect and refract when it encounters an interface between two media (denoted as 1 and 2) with contrasting acoustic impedance, which is governed by the well-known Snell's law as follows

$$
\frac{\sin \theta_{i}}{\sin \theta_{t}}=\frac{c_{1}}{c_{2}}=\frac{n_{2}}{n_{1}}
$$

where $\theta_{i}$ and $\theta_{t}$ refer to the incident and the transmitted angles respectively, $c_{p}$ and $n_{p}$ are the sound velocity and refractive index respective with the subscript $p=1,2$ referring to media air and xenon respectively. As media 1 and 2 are chosen as air and xenon, therefore, it can be expected that the propagating wave will behave differently. When the acoustic waves propagates from air to xenon, the wave is entering a medium with higher refractive index; hence there is refraction regardless of the incident angle $\theta_{i}$. The angle of refraction is smaller than $\theta_{i}$ and can be calculated as $\theta_{t}=\sin ^{-1}\left(\sin \left(\theta_{i}\right) / 2.03\right)$. In contrary, the acoustic waves transmitting from xenon to air are supposed to meet a medium with lower refractive index, and will definitely be 
blocked due to the total reflection occurring as the incident angle exceeds the critical angle $\theta_{c}=\sin ^{-1}\left(n_{1} / n_{2}\right)=29.5^{0}$

Our proposed scheme is based on rational exploitation of this simple yet effective mechanism.

For the specific configuration shown in Fig. 1(a), it would be reasonable to define the transmission direction of wave coming from the left and right sides as the "positive" direction (PD) and "negative" direction (ND) respectively. When the incident plane wave comes from left, as schematically illustrated in Fig. 1(b), one has $\theta_{i 1}=45^{\circ}$; and it can be readily predicted by the Snell's law that the transmitted angle is $\theta_{t 1}=20.4^{0}$. Considering that $\theta_{t 1}+\theta_{i 2}=45^{0}$, the incident angle of the wave that propagates within the xenon filled the polygon and then impinges on the interface at the right side will become $\theta_{i 2}=24.6^{0}$, leading to a subsequent refraction angle of $\theta_{t 2}=57.7^{0}$. This suggests that the normal incident plane wave coming from the left side is allowed to pass through the whole polygonal regions, and then leaves it along a predicted direction, as shown by the trajectories in Fig. 1(b).

However, the plane wave incident from the opposite direction will impinge on the rightmost side normally and is permitted to penetrate into the xenon filled the polygonal region without change in the propagation direction. In such a case, the incident angle on the left sides (denoted as $\left.\theta_{i 3}\right)$ will be $45^{\circ}$, which is apparently much larger than the critical angle we derived above. It is therefore expected that the acoustic waves will undergo a total reflection and cannot pass, as shown schematically in Fig. 1(c). As a result, the designed devices with a polygonal shape will allow only the transmission of acoustic waves coming along the PD while reflecting virtually the incident acoustic energy propagating along the $\mathrm{ND}$, leading to a substantially asymmetric transmission property.

Notice that in our design we use a natural material with innately dispersionless acoustical 
parameters, which offers a high refractive index absolutely independent of the driving frequency in stark contrast to the previous artificial materials that have to depend on acoustic resonances to enhance the effective refractive index and will unavoidably lose their extraordinary acoustical properties when the frequency deviates from the eigen-frequency of individual units [8]. Furthermore, the introduced gas has nearly the same mass density as air and is optically transparent, so there is no need of elaborated solid structures that increase the weight and opaqueness of the resulting device. As a consequence, our designed device can be expected to bear the advantages of ultra-broad bandwidth, ultra-light weight, total transparency and simple and low-cost fabrication.

\section{Simulations and analysis}

We have carried out a series of numerical simulations to evaluate the performance of our designed devices, by employing the finite-element method based on COMSOL Multiphysics. Typical numerical results are shown in the following figures. The spatial distributions of acoustic pressures are calculated for the waves transmitting along two opposite directions at three specific frequencies, i.e., $1500 \mathrm{~Hz}, 10 \mathrm{kHz}, 20 \mathrm{kHz}$, and are plotted in Fig. 2.

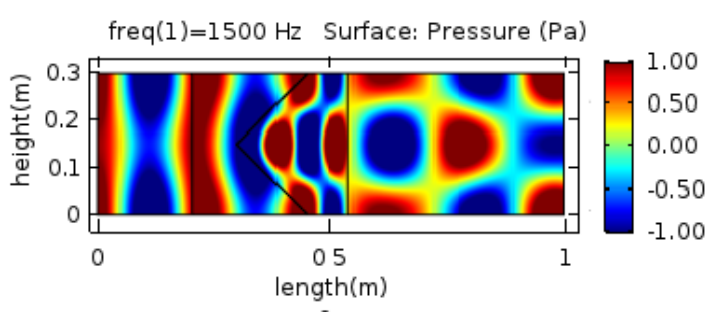

(a) $\mathrm{PD}, \mathrm{f}=1500 \mathrm{~Hz}$

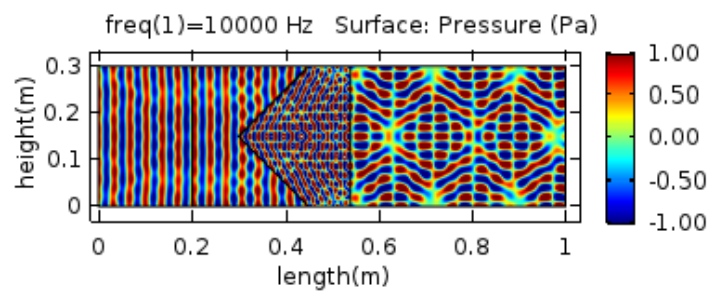

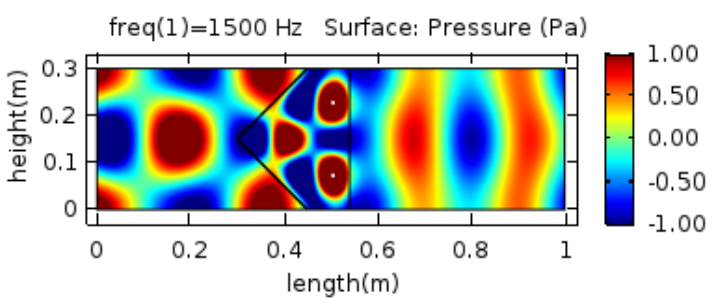

(b) $\mathrm{ND}, \mathrm{f}=1500 \mathrm{~Hz}$

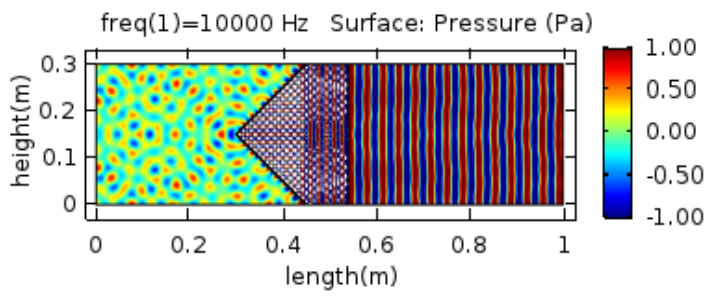


(c) $\mathrm{PD}, \mathrm{f}=10 \mathrm{kHz}$

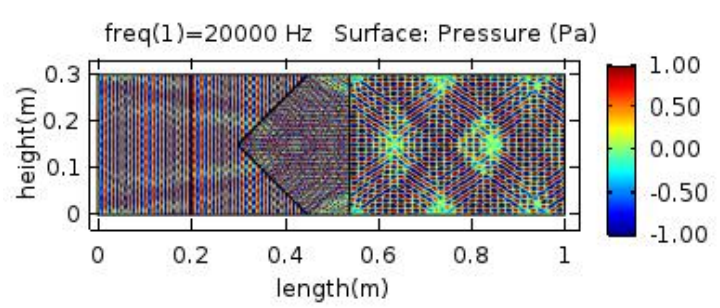

(e) $\mathrm{PD}, \mathrm{f}=20 \mathrm{kHz}$ (d) $\mathrm{ND}, \mathrm{f}=10 \mathrm{kHz}$

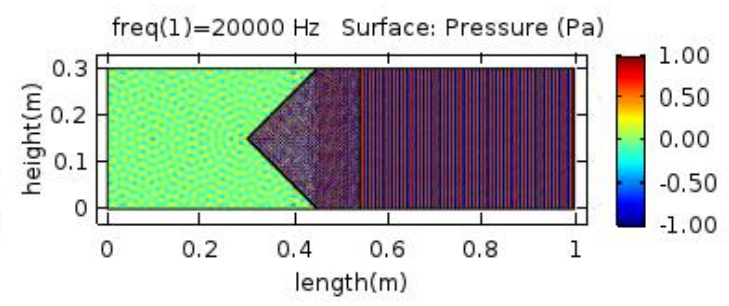

(f) $\mathrm{ND}, \mathrm{f}=20 \mathrm{kHz}$

FIG 2 Spatial distribution of the acoustic pressure generated by the incident plane waves propagating along the (a,

c, e) PD and (b, d, f) ND for three particular frequencies: (a,b) $1500 \mathrm{~Hz},(\mathrm{c}, \mathrm{d}) 10 \mathrm{kHz}$, and (e, f) $20 \mathrm{kHz}$, respectively.

To better understand the asymmetric transmission along the two different directions, Here the transmission is defined as the ratio between the intensities of the transmitted wave and in cident wave. It is observed that the transmission property of the proposed structure becomes nearly symmetric in the quasi-static limit. As the frequency of incident wave is extremely low, the typical dimensions of the polygonal region, e.g., the height and the maximum width which are chosen as $0.3 \mathrm{~m}$ and $0.24 \mathrm{~m}$ respectively as we have mentioned above, will become comparable to the operating wavelength (such as $0.23 \mathrm{~m}$ at $1500 \mathrm{~Hz}$ ), leading to strong diffraction effects at the left part of the devices which will not yield the desired total reflection since the propagating wave is not governed by geometric acoustics in such cases. As a proof, the transmissions are numerically calculated to be 0.85 and 0.69 for PD and ND at $1500 \mathrm{~Hz}$, showing that most of the incident wave energy is permitted to pass along either direction.

However, when the frequency is sufficiently high, the designed structure produce a highly asymmetric transmission for the incident plane wave travelling along the two opposite directions, as proven by the numerical results shown in Fig. 3. In the ND, the transmitted waves are blocked 
as expected by the occurrence of nearly total reflection at the interface between xenon and air on the left side, despite it being allowed to penetrate the rightmost interface as a result of normal incidence, as illustrated by Figs. 2(d) and (f). Contrarily, the plane wave propagating along the PD is able to pass through the whole polygonal region and leave it after being split into two parts. In particular, although the upper and lower parts of the wavefront of the transmitted wave overlap and lead to interference pattern in the output region of the waveguide, it can be clearly observed that these two parts of wavefront are travelling along the predesigned directions which agree quite well with the aforementioned analytical predictions obtained on the basis of the Snell's law. In the $\mathrm{PD}$, the transmission efficiency turns out to be very high (0.92 at both $1 \mathrm{kHz}$ and $20 \mathrm{kHz}$ ), in comparison to the vanishing transmission in the ND $(0.04$ and 0.01 for these two particular frequencies respectively).

Furthermore, we need to emphasize that such an asymmetric transmission should be independent of the driving frequency due to the fact that our scheme is based on a direct manipulation of the propagation trajectory for the incident wave as we discussed above. This is evidenced by the pressure fields displayed in Fig. 2 which shows that the propagation trajectories of the transmitted waves of different frequencies are always exactly the same as the results depicted in Fig. 1(c) despite the difference in their wavelengths.

To better evaluate the ultra-broadband functionality of the proposed device, we have calculated and plotted the frequency dependence of the transmissions of left- and right-going waves in Fig. 3, which clearly shows how the acoustic energy transmitted along the two opposite directions varies as a function of the driving frequency. In Fig. 3, the blue and the red lines indicate the transmission along the PD and ND respectively. In the quasi-static limit, the blue and 
red lines almost overlap, suggesting that the transmission is approximately symmetric when the frequency is extremely low, owing to the reason discussed above. With the gradual increase of the frequency, the designed polygonal structure becomes larger in terms of the wavelength, giving rise to the phenomenon of asymmetric acoustic transmission we have predicted on the basis of geometric acoustics. It can be observed that a highly asymmetric transmission persists throughout the spectrum except for the frequency region below approximately $1500 \mathrm{~Hz}$.

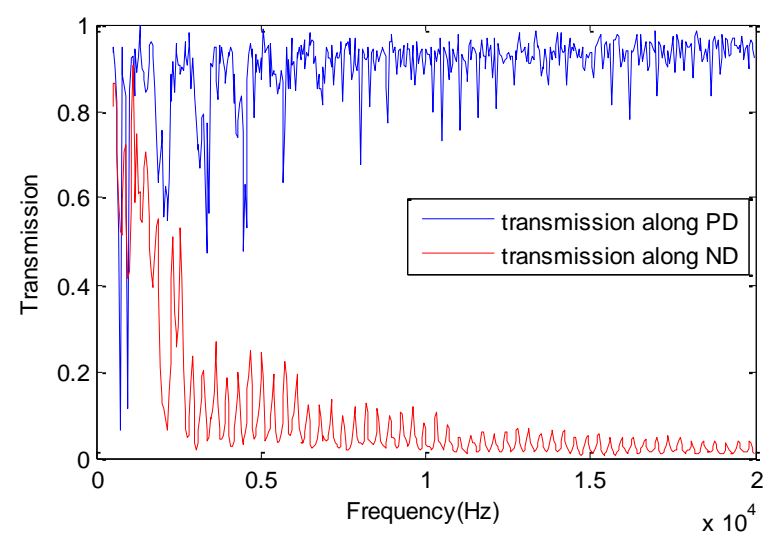

FIG 3 The frequency dependence of the transmissions along the two opposite directions. The blue and red lines indicate the transmission along the PD and ND respectively.

What deserves mentioning here is the essential difference between the ultra-broadband functionality we achieved here with innately dispersionless natural materials and that possessed by the previous metamaterial-based designs for which even the non-resonant artificial structure will still become invalid when the frequency is so high that the effective medium approximation fails. In our design, both the transmissions along the two opposite direction become more stable as the frequency exceeds $6000 \mathrm{~Hz}$ ( $>0.9$ for $\mathrm{PD}$ and $<0.1$ for ND respectively, except some wave troughs which may be caused by the reflection of hard boundaries) where geometric acoustics approximation holds quite well, and there is in principle no upper limit for such a working band. 
For the purpose of giving a quantitative estimation of the performance of asymmetric transmission, we further introduce a parameter of contrast ratio, defined as $r_{c}=\left|T_{P D}-T_{N D}\right| /\left|T_{P D}+T_{N D}\right|$, where $T_{P D}$ and $T_{N D}$ are the transmissions along the PD and ND respectively, and the corresponding numerical results are displayed in Fig. 4. The more this parameter approaches 1, the more asymmetric the transmission is. The numerical results show that the value of $r_{c}$ is extremely low in the low frequency regime below approximately $1100 \mathrm{~Hz}$ where the diffraction effect dominates, corresponding to almost identical transmission coefficients for waves transmitting along the two opposite directions, which is consistent with the results in Fig. 3. The transmission becomes more asymmetric as the frequency gradually increases, and a near-unity $r_{c}$ is observed despite slight fluctuations within a ultra-broad band with its lower limit of approximately $6000 \mathrm{~Hz}$, suggesting a high transmission efficiency along the PD while a virtually blocked transmission along the reversed direction.

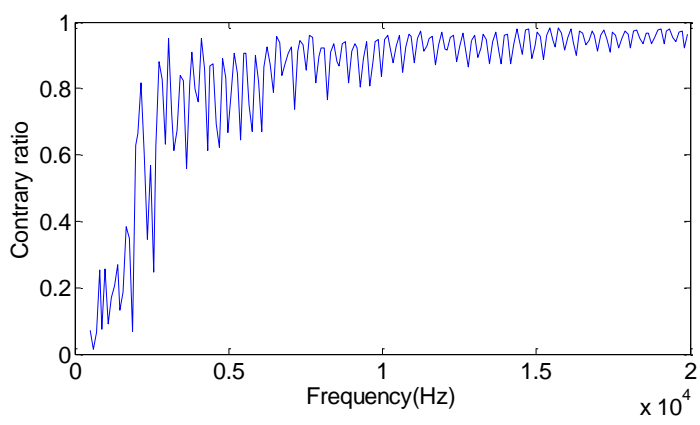

FIG 4 The frequency dependence of contrary ratio, which indicates the extent to transmission asymmetry.

The more this parameter approaches 1 , the more asymmetric the transmission is.

\section{Conclusion}

In summary, we have designed an ultra-lightweight and optically transparent structure with asymmetric transmission property. A spatial region of asymmetric shape filled with xenon is 
applied to permit the incident plane wave transmitting in the PD while blocking almost all wave that is incident in the ND in broadband. The asymmetric transmission behaves well at high frequency especially when the frequency is above $6000 \mathrm{~Hz}$, while in the quasi-static limit, the transmission in PD is quite close to that of ND because of the size of triangle comparable to wavelength. The results conclusively verify the effectiveness of the proposed design with a highly asymmetric transmission of airborne sound in an ultra-broad frequency range via numerical simulations. The realization of high efficiency and broadband asymmetric acoustic manipulation with a simple, ultra-lightweight and optically transparent structure may be highly suited for the purpose of one-way acoustic manipulation such as noise control and so on.

\section{Acknowledgement}

This work was supported by the National Natural Science Foundation of China (Grant No. 11634006).

\section{References}

1. Liang, B., Guo, X., Tu, J., Zhang, D. \& Cheng, J* An acoustic rectifier. Nature Mater. 9, 989-992 (2010).

2. Liang, B., Yuan, B. \& Cheng, J.-c.* Acoustic diode: Rectification of acoustic energy flux in one-dimensional systems. Phys. Rev. Lett. 103, 104301 (2009).

3. Zhu, X., Zou, X., Liang, B. \& Cheng, J* One-way mode transmission in one-dimensional phononic crystal plates. J. Appl. Phys. 108, 124909 (2010).

4. Li, Y., Tu, J., Liang, B*, Guo, X., Zhang, D. \& Cheng, J. Unidirectional acoustic transmission based on source pattern reconstruction. J. Appl. Phys. 112, 064504 (2012).

5. Li, R.-Q., Liang, B*, Li, Y., Kan, W.-W., Zou, X.-Y. \& Cheng, J.-C. Broadband asymmetric acoustic transmission in a gradient-index structure. Appl. Phys. Lett. 101, 263502 (2012). 
6. Li, Y., Liang, B.*, Gu, Z.-m., Zou, X.-y. \& Cheng, J.-c.* Unidirectional acoustic transmission through a prism with near-zero refractive index. Appl. Phys. Lett. 103, 053505 (2013).

7. Zhu, Y.-F., Zou, X.-Y., Liang, B.* \& Cheng, J.-c.* Broadband unidirectional transmission of sound in unblocked channel. Appl. Phys. Lett. 106, 173508 (2015).

8. Zixian Liang and Jensen Li, Extreme Acoustic Metamaterial by Coiling Up Space,Phys. Rev. Lett. 108, 114301 Published 16 March 2012 